\title{
Inhibition of the Motility of Gill Cilia of Dreissensia by Plasma of Cystic Fibrosis Patients and their Parents
}

\author{
G. T. N. BESLEY, A. D. PATRICK, and A. P. NORMAN \\ From the Institute of Child Health, Guilford Street, London W.C.1
}

\begin{abstract}
Cystic fibrosis of the pancreas is the commonest recessive disease in Britain, with a frequency of approximately 1 in 2000 live-born children (Hall and Simpkiss, 1968). A test for the heterozygous carriers of the mutant gene concerned is therefore much needed for genetic counselling. One test for heterozygotes, depending on metachromatic screening of cells in fibroblast culture, has been reported recently by Danes and Bearn (1968). A further test having possibilities of development into a routine procedure is that reported by Spock et al. (1967) who showed that whole sera and a euglobulin component isolated from the sera of patients with cystic fibrosis disrupted the normal beat of the cilia of human and rabbit respiratory tract epithelium in tissue culture. This effect was also observed with sera from parents of affected children, but was entirely absent in a group of control subjects. We supposed that this effect might be observed more generally on ciliated tissues from a wide variety of organisms, and that greater scope and simplicity might thereby be given to the laboratory investigation of this interesting finding. This report describes such observations using segments of gills of the fresh-water mussel Dreissensia as an experimental system.
\end{abstract}

\section{Methods}

Dreissensia is commonly found in European waterways and lakes, and healthy stocks may be maintained in the laboratory for several months in an adequately large volume of water, preferably that from which they were gathered. Non-fluoridated tap-water may be tolerated if the $p \mathrm{H}$ is 7.5 to $8 \cdot 0$. Circulation or aeration of the water is advisable and cool conditions must be maintained. The four whole gill plates are carefully dissected and left for 1 hour in fresh water. Segments (approximately $3 \mathrm{~mm}^{2}$ ) are then cut with scissors from the frontal borders of the gills, with a minimum of handling, and are immediately transferred to recessed glass slides containing $0.05 \mathrm{ml}$. plasma and $0.10 \mathrm{ml}$. water (in later experiments $0.05 \mathrm{ml}$. plasma $+0.30 \mathrm{ml}$. water gave

Received February 6, 1969. more satisfactory results). Up to 20 gill segments may be obtained from one large mussel, so that a number of controlled tests may be performed on the same animal. Preliminary examination of the gill segments should be made to ensure that the tissue is in good condition, as evidenced by intact structure, uniform ciliary activity, and absence of excess mucus. Sometimes, cilia were found to stop beating for a few minutes when the gill segments were first added to plasma; they then quickly resumed normal activity. This effect was presumably due to readjustment to the changed chemical or osmotic environment.

For observation of the specimens a Union inverted microscope at $\times 300$ magnification was used. When the slides were not required for observation, humidity was maintained by storage in a covered Petri dish containing moist filter paper.

Plasma from heparinized blood was usually separated immediately after collection in plastic tubes, and stored. overnight at $2^{\circ} \mathrm{C}$. before separation. Some cystic fibrosis (CF) specimens were stored at $2^{\circ} \mathrm{C}$. for several months without apparent loss of activity, while others became inactive in less than a week. After heating at $100^{\circ} \mathrm{C}$. for 2 minutes, specimens were totally inactive and were almost invariably so after freezing. It was also noted that activity decreased rapidly when plasma was stored in glassware, which perhaps indicates a basic character of the active component.

The ages of the CF patients ranged from 3 months to 16 years. Diagnostic criteria were those described by Mantle and Norman (1966). Parents of these patients were all in good general health when tested. Control plasma specimens were obtained from 11 children, aged 1 month to 14 years, admitted to hospital with a variety of illnesses; 9 asthmatic children; 12 normal healthy adults; and 9 specimens of cord blood.

\section{Results and Discussion}

Initial results obtained with plasma of $34 \mathrm{CF}$ patients and their parents (28) are compared with the controls (41) in the Fig., and have been briefly reported elsewhere (Patrick, 1968). In the CF and parents groups, the end-point time recorded for the inhibition of ciliary motility in most cases represents the time after which virtually all ciliary activity had 


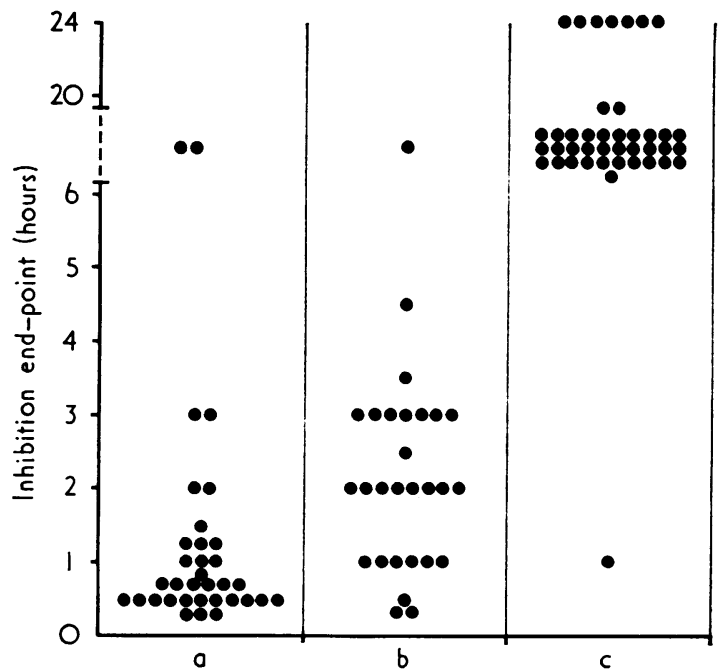

FIG. Inhibitory effect of plasma on the motility of gill cilia of Dreissensia. (a) Children with cystic fibrosis of the pancreas; (b) parents of affected children; (c) controls.

ceased. In some cases, however, small groups of lateral and latero-frontal cilia continued to beat in an uncoordinated manner for several hours further. In all cases, the time required for an obvious change to be observed, e.g. disorganization of metachronic beat, accumulation of particulate material at the frontal ciliary borders, and decreased mucus transport, was much shorter than the recorded time. It was also evident that the tissue responded to the presence of both CF and normal plasma by secreting mucus, but that this response was much greater for $\mathrm{CF}$ specimens. There were indications that the inhibitory effect of CF plasma was to some extent reversible, under the experimental conditions employed. After complete inhibition of ciliary activity for several hours, a few specimens resumed beating in a slow disorganized manner.

In the control group, the results recorded as falling between 6 and 20 hours were those of tests in which cilia were found to be unaffected after 6 hours, but which were not observed further. In tests observed for more than 20 hours, some deterioration of ciliary activity was apparent, compared with gill segments incubated in water alone, but the residual activity was generally uniform. In a total of 41 control specimens, 1 positive reaction was obtained in the case of a healthy adult female whose father also reacted positively. The mother reacted negatively. This finding of a single positive test in 41 control subjects is compatible with the theoretical heterozygote incidence of about 1 in 25 of the general population, corresponding to the incidence of homozygotes of a little over 1 in 2000 births. Both parents in 8 affected families were tested and each gave a positive reaction.

Close agreement between results of repeated tests was obtained in all but three of the total number of subjects. These were an affected child and its parent who both showed little change after 6 hours in their first tests, which were performed at the same time. Complete inhibition was observed after 1 hour and 2 hours, respectively, when these tests were repeated. Another affected child, both of whose parents gave positive reactions, reacted negatively when first tested, but showed complete inhibition after 90 minutes in a repeat test. The results of the negative tests only are given in the Fig. There was no obvious reason for these failures.

Attempts to obtain more precise assessment of the inhibitory effect of plasma using a stroboscopic method to measure the beat frequency of cilia were largely unsuccessful using Dreissensia. This difficulty resulted from the persistence of flicker image when using stroboscopic illumination at frequencies lower than about 15 per second. A few tests using the larger fresh-water mussel Anodonta showed only a weak transient inhibitory effect of CF plasma, and no comparative end-point could be determined. Tests using the sea-water mussel Mytilus failed to show any ciliary inhibition with CF plasma.

The foregoing observations using Dreissensia were made between mid-April and late September 1967. During this time, it was apparent that individual and seasonal variations of the mussels occurred, which introduced minor technical difficulties in the performance of the test. For example, some animals deteriorated very quickly after about 2 weeks in stock, and specimens swollen with eggs were generally unsuitable. In such cases deterioration was usually indicated by the increased fragility of gills, thickening of their mucus coat, and rapid impairment of ciliary activity of segments incubated in water only.

Between late September 1967 and early April 1968, which corresponded approximately to the dormant mode of the mussel, differences in the effect of CF and normal plasma could no longer be determined. This lack of differentiation was mainly due to the increased ciliostatic effect of normal plasma such that considerable overlap of the CF and normal groups occurred. The most obviously changed external feature of the mussel was the increased amount of mucus covering the gills, and it is possible that physico-chemical changes in this mucus, with consequent variation in the surface properties of the gill membranes, were responsible for the increased sensitivity to normal plasma. 
In mid-April 1968, corresponding to the renewed active growth phase of the mussel, the successful differentiation of $\mathrm{CF}$ and normal plasma was again observed and continued until the end of June, when most mussels again became sensitive to normal plasma and have remained so into the present dormant period. Attempts to overcome these changes by prolonged prewashing of the gills in water or dilute buffer solutions and by the addition of buffers to the incubation medium were unsuccessful. However, some evidence was obtained that considerable improvement might be effected by preliminary fractionation of plasma on Sephadex gel. Gills were insensitive to an albumin concentration higher than that existing in plasma, but reacted to CF total globulin fractions at higher dilutions than had been used previously for whole plasma. At these higher dilutions the overlap with normal plasma was largely eliminated.

The results presented above tend to confirm those of Spock et al. (1967) in demonstrating ciliostatic activity of cystic fibrosis plasma. If the existence of a specific association between the ciliostatic effect and the primary genetic abnormality proves to be correct, these observations may provide a basis for the development of a test useful both in diagnosis and in the detection of heterozygote frequency and distribution. However, the use of an invariable ciliated system and the determination of controllable conditions under which to operate such a system are required. The test using Dreissensia gills, though relatively crude, reliably detected both patients and their parents in our initial study, but did not maintain its reliability, under present test conditions, in succeeding trials. Nevertheless, with further experience of this and other ciliated systems, it is felt that adequate control over variability might be achieved.

\section{Summary}

In initial tests, children with cystic fibrosis of the pancreas, and their parents, were successfully differentiated from control subjects by the ciliostatic effect of their plasma on segments of gills of the fresh-water mussel Dreissensia. The incidence of this effect in the control group was compatible with the theoretical heterozygote frequency. However, due to seasonal variations of the mussel, the reliability of the test was not maintained throughout the year, and attempts to control variability have so far been unsuccessful.

We thank the Cystic Fibrosis Research Foundation Trust for financial support.

\section{REFERENCES}

Danes, B. S., and Bearn, A. G. (1968), A genetic cell marker in cystic fibrosis of the pancreas. Lancet, $1,1061$.

Hall, B. D., and Simpkiss, M. J. (1968). Incidence of fibrocystic disease in Wessex. F. med. Genet., 5, 262.

Mantle, D. J., and Norman, A. P. (1966). Life-table for cystio fibrosis. Brit. med. F., 2, 1238.

Patrick, A. D. (1968). In Cystic Fibrosis. CIBA Foundation Stud Group, No. 32, p. 14. Ed. by R. Porter and M. O'Connor Churchill, London.

Spock, A., Heick, H. M. C., Cress, H., and Logan, W. S. (1967). Abnormal serum factor in patients with cystic fibrosis of the pancreas. Pediat. Res., 1, 173. 